February 2011

version 2

\title{
COSMOLOGICAL PROPERTIES OF
} \section{A GENERIC $\mathcal{R}^{2}$-SUPERGRAVITY ${ }^{1}$}

\author{
Sergei V. Ketov ${ }^{a, b}$ and Natsuki Watanabe ${ }^{a}$ \\ a Department of Physics, Graduate School of Science, Tokyo Metropolitan \\ University, Hachioji-shi, Tokyo 192-0397, Japan \\ ${ }^{b}$ Institute for Physics and Mathematics of Universe, The University of Tokyo, \\ Kashiwa-shi, Chiba 277-8568, Japan \\ ketov@phys.se.tmu.ac.jp, watanabe-natsuki1@ed.tmu.ac.jp
}

\begin{abstract}
We investigate in detail the structure of the simplest non-trivial $F(\mathcal{R})$-supergravity model, whose $F$-function is given by a generic quadratic polynomial in terms of the scalar supercurvature $\mathcal{R}$. This toy-model admits a fully explicit derivation of the corresponding $f(R)$-gravity functions. We apply the stability requirements for selecting the physical $f(R)$-gravity functions, and discuss the phenomenological prospects of $F(\mathcal{R})$-supergravity in its application to cosmology.
\end{abstract}

\footnotetext{
${ }^{1}$ Supported in part by the Japanese Society for Promotion of Science (JSPS)
} 


\section{Introduction}

Unknown inflaton and unification of cosmological inflation with High-Energy Physics remain the outstanding problems beyond the Standard Models of elementary particles and cosmology. One of the easy ways of theoretical realization of an inflationary universe is given by popular theories of $f(R)$ gravity whose Lagrangian is a function $f(R)$ of the scalar curvature $R$ in four space-time dimensions (see refs. [1, 2] for a recent review). The use of those theories in the inflationary cosmology was pioneered by Starobinsky [3, 4].

An $f(R)$ theory of gravity is classically equivalent to the certain scalar-tensor theory of gravity $[5,6,7,8]$. In $f(R)$-gravity a dynamics of the spin-2 part of metric is not modified, but there is the extra propagating scalar field (called scalaron) given by the conformal mode of the metric (over Minkowski or (anti)de Sitter vacuum). That scalar field plays the role of inflaton in the inflationary models based on $f(R)$ gravity. The $f(R)$ gravity emerged as the phenomenological approach unrelated to any fundamental theory of gravity and without a connection to High-Energy Physics. We believe that such connection may be established via embedding and extending $f(R)$-gravity to supergravity, as the first step. Supergravity is more fundamental than gravity because supergravity is the theory of local supersymmetry, while local supersymmetry implies general covariance.

In our recent papers $[9,10,11,12,13]$ we proposed the new supergravity theory that we call $F(\mathcal{R})$-supergravity. It can be considered as the $N=1$ locally supersymmetric extension of $f(R)$ gravity. ${ }^{2}$ Supergravity is well-motivated in High-Energy Physics theory beyond the Standard Model of elementary particles. Supergravity is also the low-energy effective action of Superstrings that

is the theory of Quantum Gravity. ${ }^{3}$ Unlike the $f(R)$ theories of gravity, the $F(\mathcal{R})$ supergravity is more constrained by local supersymmetry and consistency. Moreover, the manifestly supersymmetric construction of $F(\mathcal{R})$ supergravity in superspace $[9,10,11]$ leads to a chiral action in curved $N=1$ superspace, which may be naturally stable against quantum corrections that are usually given by full superspace integrals. Our supersymmetric extension of $f(R)$ gravity is non-trivial because the $F(\mathcal{R})$ supergravity auxiliary fields do not propagate (this feature is called the auxiliary freedom [16]). Similarly to $f(R)$-gravity, the (complex) superconformal mode of the supergravity supervielbein (over Minkowski or anti-de Sitter vacuum) becomes dynamical in $F(\mathcal{R})$ supergravity. As was demonstrated in ref. [9], an $F(\mathcal{R})$ supergravity is classically equivalent to the standard $N=1$ Poincaré supergravity coupled to the dynamical chiral superfield, whose (nontrivial) Kähler potential and superpotential are dictated by a chiral (holomorphic) function. The extra dynamical chiral superfield is just the superconformal

\footnotetext{
${ }^{2}$ Another (unimodular) $F(R)$ supergravity theory was proposed in ref. [14].

${ }^{3}$ Some applications of $F(\mathcal{R})$ supergravity to Loop Quantum Gravity were given in ref. [15].
} 
mode of the supervielbein, or a complex scalaron. As was argued in ref. [9], the leading complex scalar field component of the chiral superfield (the superscalaron) may be identified with the dilaton-axion field in (non-perturbative) Superstrings/M-Theory.

The component structure of $F(\mathcal{R})$ supergravity is very complicated, and some of its general features were studied in refs. $[11,12]$. In particular, the first explicit derivation of a real bosonic function $f(R)$ out of the supergravity (holomorphic) function $F(\mathcal{R})$ was given in ref. [12]. In ref. [13] the natural embedding of the $\left(R+R^{2}\right)$-inflationary model into $F(\mathcal{R})$ supergravity was found, which gives a simple and viable realization of chaotic inflation [17] in supergravity. In this paper we further extend the results of ref. [12] to the case of $F$-function given by a generic quadratic polynomial in terms of the scalar supercurvature, and analyze all possible solutions to the corresponding bosonic $f(R)$-gravity functions. We also apply the stability requirements to select those of them which are physical.

Our paper is organized as follows. In sec. 2 we briefly recall the superspace construction of $F(\mathcal{R})$ supergravity, and provide the algebraic equations for the auxiliary fields. In sec. 3 we define our model of $F(\mathcal{R})$ supergravity, and explicitly derive the corresponding bosonic functions $f(R)$. The stability conditions are applied in sect. 4. Possible physical applications are discussed in sec. 5. Our conclusion and outlook is sec. 6 .

\section{$2 \quad F(\mathcal{R})$ supergravity and its auxiliary scalars}

A concise and manifestly supersymmetric description of supergravity is given by superspace. We refer the interested reader to the textbooks $[18,19,20]$ for details. Here we use the units $c=\hbar=1$ and $\kappa=M_{\mathrm{Pl}}^{-1}$ in terms of the (reduced) Planck mass $M_{\mathrm{Pl}}$, with the spacetime signature $(+,-,-,-)$. Our basic notation of General Relativity coincides with that of ref. [21].

The most succinct formulation of $F(\mathcal{R})$ supergravity exist in a chiral 4D, $N=1$ superspace where it is defined by the action [9]

$$
S=\int d^{4} x d^{2} \theta \mathcal{E} F(\mathcal{R})+\text { H.c. }
$$

in terms of a holomorphic function $F(\mathcal{R})$ of the covariantly-chiral scalar curvature superfield $\mathcal{R}$, and the chiral superspace density $\mathcal{E}$. The chiral $N=1$ superfield $\mathcal{R}$ has the scalar curvature $R$ as the field coefficient at its $\theta^{2}$-term. The chiral superspace density $\mathcal{E}$ (in a WZ gauge) reads

$$
\mathcal{E}=e\left(1-2 i \theta \sigma_{a} \bar{\psi}^{a}+\theta^{2} B\right)
$$

where $e=\sqrt{-g}, \psi^{a}$ is gravitino, and $B=S-i P$ is the complex scalar auxiliary field (it does not propagate in the theory (1) despite of the apparent presence of 
the higher derivatives). The theory (1) is classically equivalent to the standard $N=1$ Poincaré supergravity minimally coupled to the chiral scalar superfield, via the supersymmetric Legendre-Weyl-Kähler transform [9, 10]. The chiral scalar superfield, given by the superconformal mode of the supervielbein, becomes dynamical in a generic $F(\mathcal{R})$ supergravity.

As regards a large-scale evolution of the FRLW Universe in terms of its scale factor, it is the scalar curvature dependence of any gravitational effective action that plays the most relevant role there. Similarly, as regards any supergravitational effective action, the evolution of the FRLW scale factor is largely determined by a dependence of the gravitational superfield effective action upon the scalar supercurvature $\mathcal{R}$.

A bosonic $f(R)$ gravity action is given by $[1,2]$

$$
S_{\mathrm{f}}=\int d^{4} x \sqrt{-g} f(R)
$$

in terms of the real function $f(R)$ of the scalar curvature $R$. The relation between the master chiral superfield function $F(\mathcal{R})$ in eq. (1) and the corresponding bosonic function $f(R)$ in eq. (3) can be established by appplying the standard formulae of superspace $[18,19,20]$ and ignoring the fermionic contributions. As a result $[10,11,12]$, one gets a bosonic Lagrangian in the form

$$
(-g)^{-1 / 2} L_{\mathrm{bos}} \equiv f(R, \tilde{R} ; X, \bar{X})=F^{\prime}(\bar{X})\left[\frac{1}{3} R_{*}+4 \bar{X} X\right]+3 X F(\bar{X})+\text { H.c. }
$$

where the primes denote differentiation, and we have also introduced the notation

$$
X=\frac{\kappa}{3} B \quad \text { and } \quad R_{*}=R-\frac{i}{2} \varepsilon^{a b c d} R_{a b c d} \equiv R+i \tilde{R} .
$$

The $\tilde{R}$ does not vanish in $F(\mathcal{R})$ supergravity, and it represents an axion, the pseudo-scalar superpartner of real scalaron (inflaton) in our construction $[9,10$, $11,12,13]$.

Varying eq. (4) with respect to the complex auxiliary fields $X$ and $\bar{X}$,

$$
\frac{\partial L_{\mathrm{bos}}}{\partial X}=\frac{\partial L_{\mathrm{bos}}}{\partial \bar{X}}=0
$$

gives rise to the algebraic equations on the auxiliary fields,

$$
3 \bar{F}+X\left(4 \bar{F}^{\prime}+7 F^{\prime}\right)+4 \bar{X} X F^{\prime \prime}+\frac{1}{3} F^{\prime \prime} R_{*}=0
$$

and its conjugate

$$
3 F+\bar{X}\left(4 F^{\prime}+7 \bar{F}^{\prime}\right)+4 \bar{X} X \bar{F}^{\prime \prime}+\frac{1}{3} \bar{F}^{\prime \prime} \bar{R}_{*}=0
$$

where $F=F(X)$ and $\bar{F}=\bar{F}(\bar{X})$. The algebraic equations (7) and (8) cannot be explicitly solved for $X$ in a generic $F(\mathcal{R})$ supergravity. 
The stability conditions in $f(R)$-gravity are well known $[1,2]$, and in our notation they read

$$
f^{\prime}(R)<0
$$

and

$$
f^{\prime \prime}(R)>0
$$

The first (classical stability) condition (9) is related to the sign factor in front of the Einstein-Hilbert term (linear in $R$ ) in the $f(R)$-gravity action, and it ensures that graviton is not a ghost. The second (quantum stability) condition (10) ensures that scalaron is not a tachyon.

Being interested in the bosonic $f(R)$-gravity action that follows from eq. (1), we set both gravitino and axion to zero, which also implies $R_{*}=R$ and a real $X$.

In $F(R)$ supergravity, eq. (9) is to be replaced by a stronger condition [13],

$$
F^{\prime}(X)<0
$$

It is easy to verify that eq. (9) follows from eq. (11) because of eq. (6). Equation (11) also guarantees the classical stability of the bosonic $f(R)$ gravity embedding into the full $F(\mathcal{R})$ supergravity against small fluctuations of the axion field [13].

\section{Our toy-model}

First, we recall that the standard (pure) supergravity $[18,19,20]$ is reproduced in our approach by simply taking

$$
F^{\prime \prime}=0 \quad \text { or, equivalently, } \quad F(\mathcal{R})=f_{0}-\frac{1}{2} f_{1} \mathcal{R},
$$

with some (complex) constants $f_{0}$ and $f_{1}$, where $\operatorname{Re} f_{1}>0$. Then eqs. (7) and (8) are easily solved by

$$
X=\frac{3 f_{0}}{5\left(\operatorname{Re} f_{1}\right)}
$$

Substituting this solution back into the Lagrangian (4) yields

$$
(-g)^{-1 / 2} L_{\mathrm{bos}}=-\frac{1}{3}\left(\operatorname{Re} f_{1}\right) R+\frac{9\left|f_{0}\right|^{2}}{5\left(\operatorname{Re} f_{1}\right)} \equiv-\frac{1}{2} M_{\mathrm{Pl}}^{2} R-\Lambda
$$

where we have identified

$$
\operatorname{Re} f_{1}=\frac{3}{2} M_{\mathrm{Pl}}^{2} \quad \text { and } \quad \Lambda=\frac{-9\left|f_{0}\right|^{2}}{5\left(\operatorname{Re} f_{1}\right)}=\frac{-6\left|f_{0}\right|^{2}}{5 M_{\mathrm{Pl}}^{2}}
$$

As is clear from the above equations, the cosmological constant in the standard pure supergravity is always zero or negative, as is required by local supersymmetry. Since we are not interested in the standard supergravity, we assume that $F^{\prime \prime} \neq 0$ in what follows. 
Let's now investigate the simplest non-trivial Ansatz $\left(F^{\prime \prime}=\right.$ const. $\left.\neq 0\right)$ for the $F(R)$ supergravity function in the form

$$
F(\mathcal{R})=f_{0}-\frac{1}{2} f_{1} \mathcal{R}+\frac{1}{2} f_{2} \mathcal{R}^{2}
$$

with three coupling constants $f_{0}, f_{1}$ and $f_{2}$. We will take all of them to be real, since we will ignore this potential source of $C P$-violation in what follows. As regards the mass dimensions of the quantities introduced, we have

$$
[F]=\left[f_{0}\right]=3, \quad[R]=\left[f_{1}\right]=2, \quad \text { and } \quad[\mathcal{R}]=\left[f_{2}\right]=1
$$

The bosonic Lagrangian (4) with the function (16) reads

$$
(-g)^{-1 / 2} L_{\text {bos }}=11 f_{2} X^{3}-7 f_{1} X^{2}+\left(\frac{2}{3} f_{2} R+6 f_{0}\right) X-\frac{1}{3} f_{1} R
$$

Hence, the auxiliary field equation (6) takes the form of a quadratic equation,

$$
\frac{33}{2} f_{2} X^{2}-7 f_{1} X+\frac{1}{3} R f_{2}+3 f_{0}=0
$$

whose solution is given by

$$
X_{ \pm}=\frac{7}{3 \cdot 11}\left[\frac{f_{1}}{f_{2}} \pm \sqrt{\frac{2 \cdot 11}{7^{2}}\left(R_{\max }-R\right)}\right]
$$

where we have introduced the maximal scalar curvature (cf. refs. [22, 23])

$$
R_{\max }=\frac{7^{2}}{2 \cdot 11} \frac{f_{1}^{2}}{f_{2}^{2}}-3^{2} \frac{f_{0}}{f_{2}}
$$

Equation (20) obviously implies the automatic bound [12]

$$
R<R_{\max }
$$

It is worth mentioning that eq. (22) is better interpreted as the lower (or AdS) bound on the scalar curvature $(-R)$. For example, in our notation, a de-Sitter space has a negative (constant) scalar curvature $R_{\mathrm{dS}}<0$, whereas an anti-deSitter space has a positive (constant) scalar curvature, $R_{\mathrm{AdS}}>0$. It is therefore natural to demand $R_{\max }>0$ (or, equivalently, $-R_{\max }<0$ ), in order to allow a flat space $(R=0)$ too. It yields

$$
198 f_{0} f_{2}<\left(7 f_{1}\right)^{2}
$$

Substituting the solution (20) back into eq. (18) yields the corresponding $f(R)$-gravity Lagrangian (after a tedious but straightforward calculation) with

$$
\begin{aligned}
f_{ \pm}(R)= & \frac{2 \cdot 7}{11} \frac{f_{0} f_{1}}{f_{2}}-\frac{2 \cdot 7^{3}}{3^{3} \cdot 11^{2}} \frac{f_{1}^{3}}{f_{2}^{2}} \\
& -\frac{19}{3^{2} \cdot 11} f_{1} R \mp \sqrt{\frac{2}{11}}\left(\frac{2^{2}}{3^{3}} f_{2}\right)\left(R_{\max }-R\right)^{3 / 2}
\end{aligned}
$$


Expanding eq. (24) into power series of $R$ yields

$$
f_{ \pm}(R)=-\Lambda_{ \pm}-a_{ \pm} R+b_{ \pm} R^{2}+\mathcal{O}\left(R^{3}\right)
$$

whose coefficients are given by

$$
\begin{gathered}
\Lambda_{ \pm}=\frac{2 \cdot 7}{3^{2} \cdot 11} f_{1}\left(R_{\max }-\frac{7^{2}}{2 \cdot 3 \cdot 11} \frac{f_{1}^{2}}{f_{2}^{2}}\right) \pm \sqrt{\frac{2}{11}}\left(\frac{2^{2}}{3^{3}} f_{2}\right) R_{\max }^{3 / 2} \\
a_{ \pm}=\frac{19}{3^{2} \cdot 11} f_{1} \mp \sqrt{\frac{2}{11} R_{\max }}\left(\frac{2}{3^{2}} f_{2}\right)
\end{gathered}
$$

and

$$
b_{ \pm}=\mp \sqrt{\frac{2}{11 R_{\max }}}\left(\frac{f_{2}}{2 \cdot 3^{2}}\right)
$$

Those equations greatly simplify when $f_{0}=0$. One finds [12]

$$
f_{ \pm}^{(0)}(R)=\frac{-5 \cdot 17 M_{\mathrm{Pl}}^{2}}{2 \cdot 3^{2} \cdot 11} R+\frac{2 \cdot 7}{3^{2} \cdot 11} M_{\mathrm{Pl}}^{2}\left(R-R_{\max }\right)\left[1 \pm \sqrt{1-R / R_{\max }}\right]
$$

where we have chosen

$$
f_{1}=\frac{3}{2} M_{\mathrm{Pl}}^{2}
$$

in order to get the standard normalization of the Einstein-Hilbert term that is linear in $R$. Then, in the limit $R_{\max } \rightarrow+\infty$, both functions $f_{ \pm}^{(0)}(R)$ reproduce General Relativity. In another limit $R \rightarrow 0$, one finds a vanishing or positive cosmological constant [12],

$$
\Lambda_{-}^{(0)}=0 \quad \text { and } \quad \Lambda_{+}^{(0)}=\frac{2^{2} \cdot 7}{3^{2} \cdot 11} M_{\mathrm{Pl}}^{2} R_{\max }
$$

\section{Stability conditions}

The stability conditions are given by eqs. (9), (10) and (11), while the 3rd condition implies the 2 nd one (sec. 2). In our case (24) we have

$$
f_{ \pm}^{\prime}(R)=-\frac{19}{3^{2} \cdot 11} f_{1} \pm \sqrt{\frac{2}{11}}\left(\frac{2}{3^{2}} f_{2}\right) \sqrt{R_{\max }-R}<0
$$

and

$$
f_{ \pm}^{\prime \prime}(R)=\mp\left(\frac{f_{2}}{3^{2}}\right) \sqrt{\frac{2}{11\left(R_{\max }-R\right)}}>0
$$

while eqs. (11), (16) and (20) yield

$$
\pm \sqrt{\frac{2 \cdot 11}{7^{2}}\left(R_{\max }-R\right)}<\frac{19}{2 \cdot 7} \frac{f_{1}}{f_{2}}
$$


It follows from eq. (33) that

$$
f_{2}^{(+)}<0 \quad \text { and } \quad f_{2}^{(-)}>0
$$

Then the stability condition (10) is obeyed for any value of $R$.

As regards the (-)-case, there are two possibilities depending upon the sign of $f_{1}$. Should $f_{1}$ be positive, all the remaining stability conditions are automatically satisfied, ie. in the case of both $f_{2}^{(-)}>0$ and $f_{1}^{(-)}>0$.

Should $f_{1}$ be negative, $f_{1}^{(-)}<0$, we find that the remaining stability conditions (32) and (34) are the same, as they should, while they are both given by

$$
R<R_{\max }-\frac{19^{2}}{2^{3} \cdot 11} \frac{f_{1}^{2}}{f_{2}^{2}}=-\frac{3 \cdot 5}{2^{3} \cdot 11} \frac{f_{1}^{2}}{f_{2}^{2}}-3^{2} \frac{f_{0}}{f_{2}} \equiv R_{\max }^{\text {ins }}
$$

As regards the $(+)$-case, eq. (34) implies that $f_{1}$ should be negative, $f_{1}<0$, whereas then eqs. (32) and (34) result in the same condition (36) again.

Since $R_{\max }^{\text {ins }}<R_{\max }$, our results imply that the instability happens before $R$ reaches $R_{\max }$ in all cases with negative $f_{1}$.

As regards the particularly simple case (29), the stability conditions allow us to choose the lower sign only. ${ }^{4}$

A different example arises with a negative $f_{1}$. When choosing the lower sign (ie. a positive $f_{2}$ ) for definiteness, we find

$$
\begin{aligned}
f_{-}(R)= & -\frac{2 \cdot 7}{11} f_{0}\left|\frac{f_{1}}{f_{2}}\right|+\frac{2 \cdot 7^{3}}{3^{3} \cdot 11^{2}}\left|\frac{f_{1}^{3}}{f_{2}^{2}}\right| \\
& +\frac{19}{3^{2} \cdot 11}\left|f_{1}\right| R+\sqrt{\frac{2}{11}}\left(\frac{2^{2}}{3^{3}} f_{2}\right)\left(R_{\max }-R\right)^{3 / 2}
\end{aligned}
$$

Demanding the standard normalization of the Einstein-Hilbert term in this case implies

$$
R_{\max }=\frac{3^{4} \cdot 11}{2^{3} f_{2}^{2}}\left(\frac{M_{\mathrm{Pl}}^{2}}{2}+\frac{19}{3^{2} \cdot 11}\left|f_{1}\right|\right)^{2}
$$

where we have used eq. (27). It is easy to verify by using eq. (26) that the cosmological constant is always negative in this case, and the instability bound (36) is given by

$$
R_{\max }^{\text {ins }}=\frac{3^{4} \cdot 11 M_{\mathrm{Pl}}^{2}}{2^{3} f_{2}^{2}}\left(\frac{M_{\mathrm{Pl}}^{2}}{2^{2}}+\frac{19\left|f_{1}\right|}{3^{2} \cdot 11}\right)<R_{\max }
$$

\footnotetext{
${ }^{4}$ The same sign was also chosen in ref. [12], though without giving an explanation.
} 


\section{Some applications}

The $f_{-}(R)$ function of eq. (24) can be rewritten to the form

$$
f(R)=\frac{7^{3}}{3^{3} \cdot 11^{2}} \frac{f_{1}^{3}}{f_{2}^{2}}-\frac{2 \cdot 7}{3^{2} \cdot 11} f_{1} R_{\max }-\frac{19}{3^{2} \cdot 11} f_{1} R+f_{2} \sqrt{\frac{2^{5}}{3^{6} \cdot 11}}\left(R_{\max }-R\right)^{3 / 2}
$$

where we have used eq. (21). There are three physically different regimes:

(i) the high-curvature regime, $R<0$ and $|R| \gg R_{\max }$. Then eq. (40) implies

$$
f(R) \approx-\Lambda_{h}-a_{h} R+c_{h}|R|^{3 / 2}
$$

whose coefficients are given by

$$
\begin{aligned}
\Lambda_{h} & =\frac{2 \cdot 7}{3^{2} \cdot 11} f_{1} R_{\max }-\frac{7^{3}}{3^{3} \cdot 11^{2}} \frac{f_{1}^{3}}{f_{2}^{2}} \\
a_{h} & =\frac{19}{3^{2} \cdot 11} f_{1} \\
c_{h} & =\sqrt{\frac{2}{11}}\left(\frac{2^{2}}{3^{3}} f_{2}\right)
\end{aligned}
$$

(ii) the low-curvature regime, $\left|R / R_{\max }\right| \ll 1$. Then eq. (40) implies

$$
f(R) \approx-\Lambda_{l}-a_{l} R
$$

whose coefficients are given by

$$
\begin{aligned}
& \Lambda_{l}=\Lambda_{h}-\sqrt{\frac{2 R_{\max }^{3}}{11}}\left(\frac{2^{2}}{3^{3}} f_{2}\right), \\
& a_{l}=a_{h}+\sqrt{\frac{2 R_{\max }}{11}}\left(\frac{2}{3^{2}} f_{2}\right)=a_{-}=\frac{M_{\mathrm{Pl}}^{2}}{2},
\end{aligned}
$$

where we have used eq. (27).

(iii) the near-the-bound regime (assuming that no instability happens before it), $R=R_{\max }+\delta R, \delta R<0$, and $\left|\delta R / R_{\max }\right| \ll 1$. Then eq. (40) implies

$$
f(R) \approx-\Lambda_{b}+a_{b}|\delta R|+c_{b}|\delta R|^{3 / 2}
$$

whose coefficients are

$$
\begin{aligned}
\Lambda_{b} & =\frac{1}{3} f_{1} R_{\max }-\frac{7^{3}}{3^{3} \cdot 11^{2}} \frac{f_{1}^{3}}{f_{2}^{2}} \\
a_{b} & =a_{h} \\
c_{b} & =\sqrt{\frac{2}{11}}\left(\frac{2^{2}}{3^{3}} f_{2}\right)
\end{aligned}
$$


The cosmological dynamics may be either directly derived from the gravitational equations of motion in the $f(R)$-gravity with a given function $f(R)$, or just read off from the form of the corresponding scalar potential of a scalaron (see below). For example, as was demonstrated in ref. [12] for the special case $f_{0}=0,{ }^{5}$ a cosmological expansion is possible in the regime (i) towards the regime (ii), and then, perhaps, to the regime (iii) unless an instability occurs.

However, one should be careful since our toy-model (16) does not pretend to be viable in the low-curvature regime, eg., for the present Universe. Nevertheless, if one wants to give it some physical meaning there, by identifying it with General Relativity, then one should also fine-tune the cosmological constant $\Lambda_{l}$ in eq. (44) to be "small" and positive. We find that it amounts to

$$
R_{\max } \approx \frac{3^{4} \cdot 7^{2} \cdot 11}{2^{5} \cdot 19^{2}} \frac{M_{\mathrm{Pl}}^{4}}{f_{2}^{2}} \equiv R_{\Lambda=0}
$$

with the actual value of $R_{\max }$ to be "slightly" above of that bound, $R_{\max }>R_{\Lambda=0}$. It is also posssible to have the vanishing cosmological constant, $\Lambda_{l}=0$, when choosing $R_{\max }=R_{\Lambda=0}$. It is worth mentioning that it relates the values of $R_{\max }$ and $f_{2}$.

As is well known [5, 6, 7, 24, 25], an $f(R)$ gravity theory (3) is classically equivalent to a scalar-tensor gravity having the action

$$
S\left[g_{\mu \nu}, \phi\right]=\int d^{4} x \sqrt{-g}\left\{\frac{-R}{2 \kappa^{2}}+\frac{1}{2} g^{\mu \nu} \partial_{\mu} \phi \partial_{\nu} \phi-V(\phi)\right\}
$$

in terms of the scalaron field $\phi(x)$ with the scalar potential $V(\phi)$. The equivalence is established via a Legendre-Weyl transform $[5,7,25]$. In our notation we have ${ }^{6}$

$$
f(R)=Z\left(e^{y}\right)-R e^{y}, \quad R=Z^{\prime}\left(e^{y}\right), \quad-f^{\prime}(R)=e^{y}, \quad y=\sqrt{\frac{2}{3}} \frac{\phi}{M_{\mathrm{Pl}}}
$$

so that the scalar potential is given by [25]

$$
V(y)=-\frac{1}{2} M_{\mathrm{Pl}}^{2} e^{-2 y} Z\left(e^{y}\right)
$$

It is worth noticing that the stability condition (9) implies an invertibility of the 3rd equation (49), ie. $R=R(y)$.

For instance, the simplest Starobinsky model of chaotic inflation $[3,13]$ corresponds to

$$
f_{S}(R)=-\frac{1}{2} M_{\mathrm{Pl}}^{2}\left(R-\frac{R^{2}}{6 M^{2}}\right)
$$

\footnotetext{
${ }^{5}$ See also ref. [13] for another example.

${ }^{6}$ Compared to ref. [25], we changed here $y \rightarrow-y$.
} 
where the mass parameter $M$ coincides with the inflaton mass. The corresponding inflaton scalar potential (50) is given by [24, 25]

$$
V(y)=V_{0}\left(e^{-y}-1\right)^{2}
$$

where $V_{0}=\frac{3}{4} M_{\mathrm{Pl}}^{2} M^{2}$. The constant term in eq. (52) is the vacuum energy that drives inflaton towards the minimum of its scalar potential (so that the inflation has an end). In terms of the equivalent scalar-tensor gravity (48) with the scalar ponential (52) the standard slow-roll parameters [24] are given by [25]

$$
\varepsilon=\frac{1}{2} M_{\mathrm{Pl}}^{2}\left(\frac{V^{\prime}}{V}\right)^{2}=\frac{4 e^{-2 y}}{3\left(e^{-y}-1\right)^{2}}=\frac{3}{4 N_{e}^{2}}+\mathcal{O}\left(\frac{\ln ^{2} N_{e}}{N_{e}^{3}}\right)
$$

and

$$
\eta=M_{\mathrm{Pl}}^{2} \frac{V^{\prime \prime}}{V}=\frac{4 e^{-y}\left(2 e^{-y}-1\right)}{3\left(e^{-y}-1\right)^{2}}=-\frac{1}{N_{e}}+\frac{3 \ln N_{e}}{4 N_{e}^{2}}+\frac{5}{4 N_{e}^{2}}+\mathcal{O}\left(\frac{\ln ^{2} N_{e}}{N_{e}^{3}}\right)
$$

where the primes denote the derivatives with respect to the inflaton field $\phi$, and the e-foldings number $N_{e}$ reads

$$
N_{e}=\int_{t}^{t_{\text {end }}} H d t \approx \frac{1}{M_{\mathrm{Pl}}^{2}} \int_{\phi_{\mathrm{end}}}^{\phi} \frac{V}{V^{\prime}} d \phi \approx \frac{3}{4}\left(e^{y}-y\right)-1.04
$$

The theoretical values of the CMB spectral indices [24],

$$
n_{s}=1+2 \eta-6 \varepsilon \text { and } r=16 \varepsilon \text {, }
$$

in the case of the Starobinsky model are given by [25]

$$
n_{s}=1-\frac{2}{N_{e}}+\frac{3 \ln N_{e}}{2 N_{e}^{2}}-\frac{2}{N_{e}^{2}}+\mathcal{O}\left(\frac{\ln ^{2} N_{e}}{N_{e}^{3}}\right)
$$

and

$$
r=\frac{12}{N_{e}^{2}}+\mathcal{O}\left(\frac{\ln ^{2} N_{e}}{N_{e}^{3}}\right)
$$

They agree with the old estimates [26], as well as the most recent WMAP7 data [27], when choosing $N_{e} \approx 54$ and

$$
\frac{M}{M_{\mathrm{Pl}}}=(3.5 \pm 1.2) \cdot 10^{-5}
$$

so that the Starobinsky inflationary scenario [3] is still viable.

The particular $\mathcal{R}^{2}$-supergravity model (with $f_{0}=0$ ) was introduced in ref. [12] in an attempt to get viable embedding of the Starobinsky model into $F(\mathcal{R})$ supergravity. However, it failed because, as was found in ref. [12], the higherorder curvature terms cannot be ignored in eq. (29), ie. the $R^{n}$-terms with $n \geq 3$ 
are not small enough against the $R^{2}$-term. ${ }^{7}$ The most general Ansatz (16), which is merely quadratic in the supercurvature, does not help for that purpose either.

For example, the full $f(R)$-gravity function $f_{-}(R)$ in eq. (29), which we derived from our $\mathcal{R}^{2}$-supergravity, gives rise to the inflaton scalar potential

$$
V(y)=V_{0}\left(11 e^{y}+3\right)\left(e^{-y}-1\right)^{2}
$$

where $V_{0}=\left(3^{3} / 2^{6}\right) M_{\mathrm{Pl}}^{4} / f_{2}^{2}$. The corresponding inflationary parameters

$$
\varepsilon(y)=\frac{1}{3}\left[\frac{e^{y}\left(11+11 e^{-y}+6 e^{-2 y}\right)}{\left(11 e^{y}+3\right)\left(e^{-y}-1\right)}\right]^{2} \geq \frac{1}{3}
$$

and

$$
\eta(y)=\frac{2}{3} \frac{\left(11 e^{y}+5 e^{-y}+12 e^{-2 y}\right)}{\left(11 e^{y}+3\right)\left(e^{-y}-1\right)^{2}} \geq \frac{2}{3}
$$

are not small enough for matching the WMAP observational data. A solution to this problem was found in ref. [13] by adding to eq. (16) an extra term that is cubic in the supercurvature, with a large dimensionless coefficient.

\section{Conclusion}

The purpose of this paper was to introduce the new approach to the cosmological model building based on $F(\mathcal{R})$ supergravity, develop the techniques of deriving $f(R)$-gravity from $F(\mathcal{R})$ supergravity, and apply them to the simplest example (16) with $F^{\prime \prime}(\mathcal{R})=$ const. $\neq 0$. Our choice (16) of the $F$-function was not physically motivated but was dictated by technical simplicity only. Already the simplest example (16) reveals several important new features that are superior to those of the usual supergravity characterized by $F^{\prime \prime}(\mathcal{R})=0$, namely,

- not any $f(R)$-gravity is extendable to $F(\mathcal{R})$ supergravity; a simple (polynomial) choice of the $F(\mathcal{R})$-function leads to a complicated (non-polynomial) bosonic $f(R)$-function;

- it is easy to get a positive cosmological constant, see eg., eq. (31); it may have physical applications to (primordial) dark energy;

- the natural appearance of the (AdS) bound on the scalar curvature, resembling the Special Relativity bound on the physical speed, and similar to the Born-Infeld bound on the maximal values of the electro-magnetic field strength;

\footnotetext{
${ }^{7}$ The possibility of destabilizing the Starobinsky inflationary scenario by the terms with higher powers of the scalar curvature, in the context of $f(R)$-gravity, was noticed earlier in refs. [28, 29].
} 
- the existence of instabilities;

- removing the obstacles for a simple and natural (F-term-type) realization of the cosmological inflation in supergravity [13].

As regards the last statement, some comments are in order. As is well known [24], there is a generic problem of realizing inflation in supergravity, the socalled $\eta$-problem [30]. To avoid the $\eta$-problem, one usually assumes that the Kähler potential of the chiral matter does not depend upon some chiral superfields (called flat directions), while the inflaton is supposed to be associated with one of the flat directions [31,32]. Though it is possible to realize inflation that way, the mechanism is apparently non-geometrical and requires extra fields beyond supergravity. On the other side, the $f(R)$-gravity realization of inflation is easy and geometrical [1,2], there exist its very economical formulation [3], while it can also be extended to $F(\mathcal{R})$ supergravity [13].

Since $F(\mathcal{R})$ supergravity is classically equivalent to some standard chiral matter-coupled supergravity [9], one may wonder, how $F(R)$ supergravity avoids the $\eta$-problem? The basic explanation is that the Kähler potential, which follows from $F(\mathcal{R})$ supergravity, appears to be non-trivial (ie. is given by a non-linear sigma-model), whereas its naive (free) form was one of the main assumptions used in the $\eta$-problem derivation [30].

Moreover, it is difficult to analyze the stability conditions and compute the spectral indices of inflation in any matter-coupled supergravity model with a non-trivial Kähler potential of matter. ${ }^{8}$ Therefore, the possibility of a relatively simple analysis on the $F(\mathcal{R})$ supergravity side is yet another advantage of our approach.

Both the Legendre-Weyl transform in $f(R)$ gravity and the Legendre-WeylKähler transform in $F(\mathcal{R})$-supergravity, needed to prove their equivalence to the scalar-tensor gravity (quintessence) and the standard matter-coupled supergravity, respectively, apply at the classical level, while they are likely to fail at the quantum level, because of the non-renormalizability on the quantum field theories on both sides. Revealing the (quantum) origin of the higher-order scalar supercurvature terms in the supergravity function $F(\mathcal{R})$ (say, from some fundamental theory of Quantum Gravity, like Superstrings/M-theory) is beyond the scope of this paper and beyond our ability at present.

We do not exclude possible applications of $F(\mathcal{R})$ supergravity to the present Universe and the present Dark Energy, by choosing an appropriate function $F(\mathcal{R})$. However, one needs more physical reasoning for applying supergravity to a lowcurvature cosmology. In its turn, it requires knowing more details about supersymmetry breaking. The example (16) considered in this paper does not have the correct Newton limit, unless the first and last terms in eq. (16) are negligible.

\footnotetext{
${ }^{8}$ See, however, a recent limited analysis in ref. [33].
} 


\section{Acknowledgements}

One of the authors (SVK) is grateful to H. Abe, L. Amendola, G. Dvali, A. De Felice, A. Hebecker, S. Hellerman, T. Kobayashi, K.-I. Maeda, S. Mukohyama, M. Sasaki, A.A. Starobinsky, S. Tsujikawa and J. Yokoyama for discussions.

\section{References}

[1] T. P. Sotiriou, V. Faraoni, Rev. Mod. Phys. 82 (2010) 451, arXiv:0805.1726 [hep-th]

[2] A. De Felice and S. Tsujikawa, Living Rev. Rel. 13, 3 (2010); arXiv:1002.4928 [hep-th]

[3] A. A. Starobinsky, Phys. Lett. B91 (1980) 99

[4] L.A. Kofman, A.D. Linde and A.A. Starobinsky, Phys. Lett. B157 (1985) 361

[5] B. Whitt, Phys. Lett. B145 (1984) 176

[6] J. D. Barrow and S. Cotsakis, Phys. Lett. B214 (1988) 515

[7] K.-I. Maeda, Phys. Rev. D39 (1989) 3159

[8] E. Dyer and K. Hinterbichler, Phys. Rev. D79 (2009) 024028, arXiv:0809.4033 [gr-qc]

[9] S. James Gates, Jr., and S. V. Ketov, Phys. Lett. B674 (2009) 59, arXiv:0901.2467[hep-th]

[10] S. V. Ketov, Class. and Quantum Grav. 26 (2009) 135006, arXiv:0903.0251 [hep-th]

[11] S. V. Ketov, $F(R)$ supergravity, AIP Conf. Proc. 1241, 613 (2010); arXiv:0910.1165 [hep-th]

[12] S.V. Ketov, Phys. Lett. B692, 272 (2010); arXiv:1005.3630 [hep-th]

[13] S.V. Ketov and A.A. Starobinsky, Embedding $\left(R+R^{2}\right)$-Inflation into Supergravity, arXiv:1011.0240 [hep-th]; to appear in Phys. Rev. D (2011)

[14] H. Nishino and S. Rajpoot, Phys. Lett. B687 (2010) 382

[15] S. James Gates, Jr., S. V. Ketov and N. Yunes, Phys. Rev. D80 (2009) 065003, arXiv:0906.4978 [hep-th] 
[16] S. James Gates, Jr., Phys. Lett. B365 (1996) 132 [hep-th/9508153], and Nucl. Phys. B485 (1997) 145 [hep-th/9606109]

[17] A.D. Linde, Phys. Lett. B129 (1983) 177

[18] S. James Gates, Jr., M. T. Grisaru, M. Roček and W. Siegel, Superspace or 1001 Lessons in Supersymmetry, Benjamin-Cummings Publ. Company, 1983

[19] J. Wess and J. Bagger, Supersymmetry and Supergravity, Princeton University Press, 1992

[20] I. L. Buchbinder and S. M. Kuzenko, Ideas and Methods of Supersymmetry and Supergravity, IOP Publishers, 1998

[21] L.D. Landau and E.M. Lifshitz, The Classical Theory of Fields, Pergamon Press, Oxford, 2002

[22] S. V. Ketov, Many faces of Born-Infeld theory, invited talk at the 7th Intern. Wigner Symposium, August 2001, Maryland, USA; hep-th/0108189

[23] S. James Gates, Jr., and S. V. Ketov, Class. and Quantum Grav. 17 (2001) 3561, hep-th/0104223

[24] A.R. Liddle, D.H. Lyth, Cosmological Inflation and Large-scale Structure, Cambridge University Press, Cambridge, 2000

[25] S. Kaneda, S. V. Ketov and N. Watanabe, Mod. Phys. Lett. A25 (2010) 2753, arXiv:1001.5118 [hep-th]

[26] V.F. Mukhanov, G.V. Chibisov, JETP Lett. 33 (1981) 532;

A.A. Starobinsky, Sov. Astron. Lett. 9 (1983) 302

[27] E. Komatsu et al., Seven-Year Wilkinson Microwave Anisotropy Probe (WMAP) Observations: Cosmological Interpretation, arXiv:1001.4538v3

[28] K.-I. Maeda, Phys. Rev. D37 (1988) 858

[29] A.L. Berkin and K.-I. Maeda, Phys. Lett. B245 (1990) 348

[30] H. Murayama, H. Suzuki, T. Yanagida and J. Yokoyama, Phys. Rev. D50 (1994) 2356, arXiv:hep-ph/9311326

[31] M. Kawasaki, M. Yamaguchi and T. Yanagida, Phys. Rev. Lett. 85 (2000) 3572, arXiv:hep-ph/0004243

[32] R. Kallosh and A. Linde, JCAP 1011 (2010) 011, arXiv:1008.3375 [hep-th]

[33] R. Kallosh, A. Linde and T. Rube, General inflaton potentials in supergravity, arXiv:1011.5945 [hep-th]. 